\title{
Adipose Tissue-derived Mesenchymal Stem Cells as a Strategy to Improve Recovery After Stroke
}

María Gutiérrez-Fernández* ${ }^{\square}$, Laura Otero-Ortega*, Jaime Ramos-Cejudo, Berta Rodríguez-Frutos, Blanca Fuentes, Exuperio Díez-Tejedor

*These authors contributed equally to this work.

\section{Affiliations:}

Department of Neurology and Stroke Center, Neuroscience and Cerebrovascular Research Laboratory, La Paz University Hospital, Neuroscience Area of IdiPAZ Health Research Institute, Autónoma University of Madrid, Madrid, Spain

\section{$\square$ Corresponding author}

Prof. Exuperio Díez-Tejedor, MD, PhD, FAHA, FESO

María Gutiérrez-Fernández, PhD

Department of Neurology and Stroke Centre

Neuroscience and Cerebrovascular Research Laboratory

La Paz University Hospital, IdiPAZ Health Research Institute

Autonoma University of Madrid

Paseo de la Castellana 261, 28046, Madrid, Spain

E-mail: exuperio.diez@salud.madrid.org; mgutierrezfernandez@salud.madrid.org 


\section{Abstract}

Introduction: Based on the positive results observed in experimental animal models, adipose tissue-derived mesenchymal stem cells (AD-MSCs) constitute a promising therapy for stroke treatment. However, several aspects need to be clarified to identify the optimal conditions for successful clinical translation.

Areas covered: This review focuses on AD-MSC treatment for ischemic and hemorrhagic stroke in experimental animal models. In addition, we will explore the optimization of treatment conditions including AD-MSC production, administration routes and therapeutic windows for their appropriate use in patients, and we will provide an update on clinical trials on this therapy.

Expert opinion: Compared with other cell types, AD-MSCs have been less investigated in stroke studies. Currently, experimental animal models have shown safety and efficacy with this treatment after stroke. Due to several advantages of AD-MSCs, such as their abundance and accessibility, they can be considered a promising strategy for use in patients. However, many questions are still to be resolved regarding their mechanisms of action, immune system modulation and the effects of AD-MSCs on all components of the brain that may be affected after ischemic and hemorrhagic strokes.

KEYWORDS: Adipose tissue, Hemorrhagic Stroke, Ischemic Stroke, Mesenchymal Stem Cells. 


\section{Introduction}

Worldwide, stroke is the second highest cause of death and the leading cause of disability, [1] and its burden is increasing as a consequence of a longer life expectancy. Stroke can be classified into two types: ischemic (80-87\%) and hemorrhagic (13-20\%) [2]. Ischemic stroke occurs when the cerebral blood flow (CBF) decreases as a result of an obstruction, such as a thrombus or embolism. Currently, only IV recombinant tissue plasminogen activator (rtPA) is approved for the treatment of ischemic stroke; however, its narrow therapeutic window is just 4.5 hours from symptom onset, thus few stroke patients can benefit from this treatment. Hemorrhagic strokes are caused by the rupture of a brain blood vessel or by anomalies in the cerebral vascular structure. To date, hemostasis, management of blood pressure and intracranial pressure and surgical techniques are the therapeutic approaches for hemorrhagic strokes [3].

Cell-based therapy has emerged as a promising strategy for the treatment of ischemic and hemorrhagic stroke. This approach has demonstrated beneficial effects in improving functional outcome through mechanisms implicated in brain plasticity such as gliagenesis, oligodendrogenesis, remyelination, synaptic plasticity, axonal sprouting, and angiogenesis. Various cell types have been used: embryonic stem cells (ESC), neural stem cells (NSC), hematopoietic stem cells (HSC), mesenchymal stem cells (MSCs) and also induced pluripotent stem cells (iPS). Of all of them, MSCs are particularly good candidates for cell therapy after stroke as they are easy to obtain and due to their relatively low immunogenicity. Compared to other cell types, adipose tissue-derived mesenchymal stem cells (AD-MSCs) present several 
advantages in clinical applications for neurological disorders [4]. AD-MSCs are derived from adipose tissue and thus are abundant, accessible and easy to obtain using lipoaspiration techniques. Moreover, they provide proliferation and differentiation potential [5] without adverse side effects [6-8] and they can be administered without ethical concerns. All of these advantages mean that AD-MSCs present a great opportunity for the treatment of diseases such as stroke.

In this review we focus on AD-MSC treatment for ischemic and hemorrhagic stroke in experimental animal models and we explore the optimization of this treatment and AD-MSC production, as well provide as an update on clinical trials on this therapy.

\section{AD-MSC therapy in experimental animal models of ischemic stroke}

Brain protection and repair responses are activated as a continuum soon after cerebral ischemia induction and both responses can be modulated by cell therapy [9]. The available data from experimental animal models confirm that AD-MSC administration induces improvements in functional recovery after ischemic stroke $[7,10-12]$. This functional improvement in the animals is associated with a reduction in infarct volume [12] and interestingly, when a reduction in this parameter is not found [7, 11], a degree of tissue preservation is observed that includes a reduction in global cell death (both apoptosis and necrosis) and also a reduction in reactive gliosis [7]. More specifically, AD-MSC modulation of Bcl-2-mediated apoptosis signaling and proapoptotic caspase-12 expression has been described [13].

Regarding repair and plasticity modulation by AD-MSCs, the levels of brain repairassociated markers implicated in neurogenesis and neuronal plasticity (Doublecortin (DCX); Neurofilament (NF), oligodendrogenesis (Olig-2), synaptic plasticity (synaptophisyn (SYP), and angiogenesis (vascular endothelial growth factor (VEGF) have been shown to be modulated after AD-MSC treatment $[7,12,14]$. In addition, augmented microvessel proliferation has been found after AD-MSC treatment in the brains of animals [15]. Unpublished data from our group suggest that AD-MSCs play a role in oligodendrogenesis and white matter fiber tract repair in experimental animals with subcortical stroke. These functions are of interest as most of 
studies have not focused on the white matter component after stroke and the mechanisms of fiber tract injury [16].

Although the beneficial effects of AD-MSC therapy in experimental animal models of cerebral infarct are known, the mechanisms of action of AD-MSCs after stroke are unknown. At least three mechanisms have been proposed: (i) Transdifferentiation of the administered AD-MSCs at the injury site, (ii) AD-MSC-mediated immunomodulation, and (iii) stimulation of the brain repair response via the release of trophic factors and other signaling molecules by the AD-MSCs [8]. The potential contribution of each one of these mechanisms to functional recovery has had an impact on the experimental design of previous studies on animals, as we will discuss below.

Concerning the first mechanism, AD-MSCs can differentiate into various cell lineages [17, 18] and can express typical neural markers such as microtubule-associated protein (MAP-2) and glial acid fibrillary protein (GFAP) under differentiating conditions in vitro [12]. Early studies on experimental animals with stroke aimed to analyze the in vivo differentiation potential of AD-MSCs in the brain after intracerebral administration. Given the fact that only $2 \%$ of cells survived in the brain parenchyma, their integration into functional neural networks was not shown. For this reason, the authors attributed the functional recovery to cell-tissue interactions and AD-MSC-mediated trophic factor support [19]. It was proposed that AD-MSC cell homing in the brain was not required to elicit a good functional recovery $[8,20]$.

Subsequent studies analyzed the effects of AD-MSC administration using less invasive methods such as the IV route, confirming good results in terms of functional outcome [7, 12]. Differences in brain cell homing were found and some studies did not show cerebral implantation [7, 11], whereas other studies did observe cell implantation after IV administration when using repeated doses [12]. On the other hand, AD-MSC-mediated immunomodulation has been determined to be crucial for stroke recovery. In vitro studies show that AD-MSCS suppress the proliferation of activated blood mononuclear cells, modulating IL-6 and transforming growth factor-1 (TGF-1) levels [21]. In addition, levels of IL18, toll-like receptor-4 (TLR-4) and plasminogen inhibitor PAI-1 have been shown to be reduced by AD-MSCs [12]. Previous studies have shown that mesenchymal stem cells (MSCs) are allocated in peripheral organs such as the spleen, lungs and kidney and are cleared through the kidney after IV administration [10, 22, 23]. Whether entrapment of AD-MSCs in peripheral organs might have effects on stroke recovery should be analyzed in future studies. Given the fact that the hypothalamic-pituitary-adrenal axis is a link between the brain and peripheral 
organs and is known to be involved in immunomodulation [24], it would be interesting to analyze to what extent the peripheral allocation of cells is associated with a modulation of brain inflammation.

Regarding the release of trophic factors and other signaling molecules, in vitro studies have identified AD-MSC production of brain-derived neurotrophic factor (BDNF), VEGF [25] and TGFb [26]. VEGF administration reduces infarct volume and brain edema after temporary middle cerebral artery occlusion (MCAO) [27]. Moreover, in vitro studies have shown that cell-free ADMSC culture mediums enhance neuronal survival, reduce cell death and astrogliosis, enhance angiogenesis and improve functional recovery when administered to animals after stroke [26, 28].

In recent years, it has been reported that intercellular communication of MSCs is at least in part mediated by exosomes, which are small vesicles containing proteins and functional microRNAs and their use as a therapeutic strategy has been supported [29]. In fact, systemic administration of MSC-generated exosomes has been shown to induce recovery from stroke in animals [30]. Considering that there are various cell sources of MSC, such as the bone marrow, placenta or adipose tissue, it would be interesting to analyze possible differences in the therapeutic effect of exosomes derived from each of these sources. However, previous data suggest that there are no differences in efficacy when comparing IV administration of MSC derived from the bone marrow or adipose tissue [7]. As adipose tissue is especially abundant and easy to obtain, our future studies on experimental animals aim to analyze the potential of AD-MSC-derived exosomes after stroke.

\section{AD-MSC treatment in intracerebral hemorrhage animal models}

Primary $\mathrm{ICH}$ accounts for $10-20 \%$ of all strokes, thus there are considerably fewer experimental studies analyzing new therapeutic approaches for the treatment of hemorrhagic compared to ischemic stroke. However, stem-cell therapy has shown promising results in some studies using hemorrhagic animal models. Several MSC sources are available for the treatment of this disease in basic studies and all have demonstrated good results. Results include increases endogenous neurogenesis at the level of the subventricular zone and decreased apoptosis in acute [31] and chronic [32,33] experimental animal models of intracerebral hemorrhage. In addition, anti-inflammatory, antifibrotic, antioxidative paracrine, and regenerative effects were found after intraventricular hemorrhage in an experimental animal 
model of newborn rats [34]. Moreover, various reports are accumulating evidence that ADMSCs could be a novel therapeutic strategy for $\mathrm{ICH}$. Previous experience in acute intraventricular transplantation of rat AD-MSCs showed not only functional recovery but also decreased cell apoptosis. Furthermore, AD-MSCs were able to differentiate into neuron-like and astrocyte-like cells around the hematoma. Finally, growth factor expression, particularly VEGF, was upregulated after using AD-MSCs [35]. Another study on acute intravenous human AD-MSC administration in a rat experimental model of intracerebral hemorrhage showed functional recovery by reducing acute perihematomal edema, inflammatory cell infiltration, glial thickness and apoptosis and inducing endothelial differentiation of AD-MSCs [36].

\section{Optimization of AD-MSC treatment conditions}

\subsection{AD-MSC production for stroke}

In order to obtain AD-MSCs for stroke patients, adipose tissue can be obtained from voluntary, healthy donors (allogeneic administration). The adipose tissue is subjected to collagenase treatment, and after several centrifugations the AD-MSCs are isolated. Cells are plated in flasks and when $90 \%$ confluence is reached, they are passaged to obtain the desired clinical doses. Purified cells are usually cryopreserved until needed for administration, thus when a stroke patient is ready to receive treatment, the cells are defrosted and cultured for at least one additional passage. Usually the process takes 3 to 6 passages and phenotyping, cytogenetics, safety and sterility testing is performed at every step. In general, once a stroke patient is ready to receive treatment, at least 7 days of AD-MSC preparation are needed before they are ready to be administered (Figure 1A).

Another option is to obtain the AD-MSCs from the stroke patient (autologous administration). With this type of administration, the total preparation time including collagenase treatment, plating, harvesting, phenotyping, cytogenetic and sterility testing is substantially increased. In this case there is no need for freezing, but the time for treatment preparation is usually around 4 weeks (Figure 1B). 
In terms of costs, the procedure for obtaining AD-MSCs is expensive. AD-MSCs are extracted from the donor, isolated, planted, passaged, harvested, cryopreserved and tested before being administered to the patient. This treatment preparation is expensive because it requires specific sterile installations, equipment and material and trained, specialized personnel. However, if AD-MSC therapy is established as a conventional treatment in the future, the cost per patient could be significantly reduced. With this approach, AD-MSC preparation time would be approximately 1 day to harvest the cells and perform the quality, characterization and release testing (Figure 1C).

On the other hand, several aspects would need to be standardized to optimize the clinical setting for AD-MSC preparation. For example, it would be important to standardize the cell culture media, as some research groups use platelet lysate, Dulbecco's Modified Eagle Medium (DMEM), DMEM: F12 or Roswell Park Memorial Institute (RPMI) [37]. In addition, the usage (or not) of antibiotics and the type of vehicle to be used in AD-MSC administration must be standardized. Another important issue is to optimize injection procedures to avoid celladherence, and ready-to-use vials for administration should not be stored for more than $48 \mathrm{~h}$. All of these aspects must be considered to improve AD-MSC production and to standardize the protocols for multicenter clinical trials.

In addition, to enhance the therapeutic potential, recent advances in AD-MSC culture have been made. These include the use of three-dimensional scaffolding [38], cell programming by using biodegradable polymeric nanoparticles [39], exposure to ischemia and oxidative stress in vitro [40] and the stimulation of cells by low-energy laser stimulation [41]. These advances are promising and need to be optimized for stroke by considering the specific needs and clinical conditions of stroke.

\subsection{Type of administration, delivery route and therapeutic window}

As mentioned earlier, AD-MSC administration can be autologous (AD-MSCs from the same patient) or allogeneic (AD-MSC derived from healthy donors). The immunomodulatory and immunoprivileged properties of AD-MSC are of value for their allogeneic application, as it has been shown that AD-MSCs have low MHC Class I expression and lack MHC Class II molecules and costimulatory molecules CD40, CD80 y CD86 [42]. In addition, class I molecules may activate alloreactive $T$ cells, but with the absence of costimulatory molecules, a secondary 
signal would not engage, leaving the T cells anergic [43]. This result suggests that T cells would not be likely to induce rejection when administered to patients.

Another important aspect of cell therapy is the route of administration. As previously described, a number of delivery routes have been used in experimental stroke models, including intraventricular, intrastriatal, intra-cerebral, intra-arterial and intravenous. In terms of AD-MSCs, few studies have directly compared delivery routes. Du et al compared the therapeutic effect of the three most commonly used administration routes (intravenous, intraarterial or intra-ventricular) of AD-MSCs in a rat stroke model. Their findings suggest that compared with intra-ventricular delivery, intravascular injection allows higher dose injections with fewer invasions and it appears to be optimal with regard to therapeutic efficacy, safety and feasibility [44]. In this respect, the intravenous administration of AD-MSCs may prove to be safer and less invasive for stem cell therapy in stroke patients.

On the other hand, soon after blood flow reduction, the entire ischemic cascade is initiated and protection and repair mechanisms are activated as a continuum [45] to reduce brain damage. This cascade is why time is relevant for therapeutic interventions. Various time points are considered, depending on the expected mechanism of action: early vs. late phase administration (Figure 2). However, we must consider that in all conditions, stem cell therapy could help widen the therapeutic window of opportunities for stroke patients, which is currently limited to $4.5 \mathrm{~h}$ for rtPA.

By administering AD-MSCs as early as possible, both protection and repair mechanisms could be modulated. In our previous experience with rodents, IV administration of AD-MSCs at $30 \mathrm{~min}$ after stroke showed significant improvement in functional recovery scales even at $24 \mathrm{~h}$ and was maintained at 14 days after stroke. Moreover, we observed higher levels of brain repair-associated markers implicated in neurogenesis and neuronal plasticity (NF), oligodendrogenesis (Olig-2), synaptic plasticity (SYP), and angiogenesis (VEGF) after AD-MSC treatment $[7,10]$. Other authors have also obtained good results when administering the cells at $24 \mathrm{~h}$ after the induction of stroke [12]. Although time point relationships in animals versus humans are a subject of debate, the beneficial effects of early administration (30 min, $24 \mathrm{~h}$, etc.) are attributed to a modulation of both protection and early repair processes.

On the other hand, a number of studies in animals have also shown positive effects on brain repair stimulation (neurogenesis and angiogenesis) when administering the cells during the post-acute/chronic phase (late phase administration) [46]. Cell administration up to 1 
month after stroke showed functional recovery, reduced scar thickness, and an increased number of proliferating cells and oligodendrocyte precursor cells along the subventricular zone in the ipsilateral hemisphere [47]. However, in another study, late phase administration (1 month) did not show a functional outcome [48]. Considering that all of these studies were performed with bone marrow derived MSCs, it would be interesting to analyze this approach with AD-MSCs in future studies.

\section{Road to the clinic}

Cell therapy has emerged as a novel strategy for the treatment of a many diseases. MSCs have been used in several clinical contexts such as ischemic stroke, cardiomyopathy, myocardial infarction, Crohn's disease, and graft versus host disease, as reflected in a systematic review and meta-analysis [49]. A total of 87 clinical trials with AD-MSCs have been performed worldwide [37]. However, few studies have evaluated the safety of the administration of AD-MSCs after ischemic stroke. One study is a pilot clinical trial, phase lla, to evaluate the safety of a single IV administration of allogeneic AD-MSCs or placebo (or vehicle) administered in the first 2 weeks after the onset of stroke symptoms (NCT01678534). The other clinical trial is in phase $\mathrm{I} / \mathrm{II}$ and assesses the safety and effects of autologous AD-MSCs via internal carotid artery and IV administration (NCT01453829) [49]. These two clinical trials are designed to demonstrate the safety of AD-MSC administration, but more studies are needed that permit the inclusion of more patients, in order to assess the efficacy of AD-MSC treatment.

Regarding hemorrhagic stroke clinical trials, there are considerably fewer studies analyzing stem cell therapy for its treatment and none are using adipose tissue as a source of MSCs. However, other sources of MSCs have shown promising results for treatment in some clinical trials of hemorrhagic stroke. Autologous intracerebral transplantation of bone marrow/derived MSCs is being tested in a clinical trial to assess their safety and feasibility in patients with chronic hemorrhagic stroke (NCT01714167). Moreover, the safety of repeated IV injections of MSC derived from the umbilical cord blood will be assessed (NCT02283879). Furthermore, a phase I clinical trial on the safety and dose determination of umbilical cord blood cells will be evaluated in premature infants with intraventricular hemorrhage (NCT02274428) [49]. Even if no clinical trial is analyzing the effects of AD-MSCs in hemorrhagic stroke patients, existing trials are opening the path to prove whether MSCs could be safe as treatment for this kind of 
stroke. If these clinical trials demonstrate safety and feasibility in patients with cerebral hemorrhage with these sources of MSCs, new clinical trials could be initiated with AD-MSC treatment to confirm these results.

To date there is no evidence of negative results in terms of safety after AD-MSC administration; however it is important to consider that AD-MSCs are important contributors to the development and progression of previously existing tumors [50-52].

\section{Conclusion}

Experimental evidence suggests that AD-MSC administration can enhance recovery from ischemic and hemorrhage stroke in animal models. However, many questions are still to be resolved regarding cell production, routes of administration, the therapeutic window, mechanisms of action, and immune-modulation to improve AD-MSC treatment. From the clinical point of view, it is necessary to demonstrate the safety and efficacy of these cells in stroke patients.

\section{Expert Opinion}

As the global population ages, the incidence of stroke is increasing and continues to be a public health problem. There is a clear need for better ways to improve functional and cognitive outcomes after stroke. AD-MSC therapy is a promising approach that has shown good results in experimental animal models. However, the mechanisms of action are not yet fully understood. It is important to highlight the relevance of studying the mechanisms of ADMSC therapy and its effects on all components of the brain that may be affected after ischemic and hemorrhagic stroke. For decades, research on stem cell therapy has focused on the effects of this treatment on gray matter repair (neurogenesis, angiogenesis, sinaptogenesis, astrogeneisis, vasculogenesis). More recently, a few translational studies are beginning to highlight the importance of considering the white matter component for repair after stroke [16]. Not only are $\leq 25 \%$ of ischemic strokes in humans subcortical or lacunar and confined to white matter regions, but cortical infarcts also produce white matter injury. The high frequency of this damage motivates research into the mechanisms underlying the repair of damaged white matter (axons and myelin) after a stroke [53,54]. 
Of the mechanisms attributed to AD-MSC function, functional recovery seems to be mediated by immunomodulation and trophic factor release. In our experience, the administration of BDNF (a trophic factor secreted by AD-MSCs) results in better functional outcomes, oligodendrogenesis, remyelination and fiber connectivity, as observed in rats subjected to subcortical damage in ischemic stroke [55]. Further studies to analyze the effects of additional factors released by AD-MSCS are warranted, to better understand their mechanisms of action.

As mentioned earlier, the administration of AD-MSCs can be autologous or allogeneic. To treat patients during the acute phase of stroke, allogeneic administration would be the ideal route to reduce the time needed for cell expansion and production. Allogeneic AD-MSC administration is promising, as experimental evidence in animal models of stroke has shown enhanced recovery.

One of the most important issues in cell therapy studies in stroke is the time to AD-MSC administration. Immediately after stroke, a natural repair response is activated including both protection and repair processes that continuously communicate to reduce the injury. By administering the AD-MSCs as early as possible, these mechanisms could be modulated. For this reason, in our previous experimental animal studies, AD-MSC were administered as soon as 30 minutes after stroke. Unfortunately, human clinical conditions are quite different because the situations of stroke patients cannot be predicted. Having cryopreserved cells poses a technical problem regarding the time of administration, because to improve their viability, AD-MSCs must be thawed and cultured for at least one week prior to administration. If cell therapy were established as the usual treatment, it would be possible to continuously produce AD-MSCs and avoid the need to freeze the cells, reducing by at least one week the time that is now needed for cell preparation.

Another aspect to highlight regarding the time to AD-MSC administration is the patient status after stroke. Having shown that AD-MSC administration induces a modulation of the immune system response, depending on patient status, immune system response-mediated AD-MSCs also could present a problem of infection control. Preclinical experimental models of stroke are developed in conditions of infection control, because the animals are isolated and include healthy controls. However the patients participating in a clinical trial are not in the same conditions and they can experience infections before or after suffering a stroke. A patient with an infection should not be subjected to an immune system modulation by any 
treatment such as AD-MSC administration. This aspect must be analyzed in future studies on experimental animals and patients.

Currently, only a few studies with a limited number of patients are ongoing using AD-MSCs. In order to demonstrate their efficacy in stroke patients, an investment is needed in developing multicenter clinical trials that include a high number of patients. The results would be of help in optimizing treatment parameters and cell production.

\section{Declaration of interest}

The authors declare no conflicts of interest and have received no payment for preparation of the manuscript.

\section{Acknowledgments}

This project is supported by research grants PS12/01754 (Spanish Ministry of Science) and INVICTUS (RD12/0014/0006) (Spanish Neurovascular Network), and Research Institute Carlos III, Ministry Science and Innovation.

We would like to thank ServingMed.com for their editorial assistance.

\section{Bibliography}

1. World Health Organization. The top 10 causes of death - Homepage [http://www.who.int/mediacentre/factsheets/fs310/en/]

2. Albertson M, Sharma J. Stroke: current concepts. S D Med 2014; 67: 457-61.

3. Qureshi A, Mendelow D, Hanley D. Intracerebral haemorrhage. Lancet 2009; 373: $1632-44$

4. Chang KA, Lee JH, Suh YH. Therapeutic potential of human adipose-derived stem cells in neurological disorders. J Pharmacol Sci 2014; 126:293-301

5. Parker AM, Katz AJ. Adipose-derived stem cells for the regeneration of damaged tissues. Expert Opin Biol Ther 2006; 6:567-78.

6. Ra JC, Shin IS, Kim SH, et al. Safety of intravenous infusion of human adipose tissuederived mesenchymal stem cells in animals and humans. Stem Cells Dev 2011; 20:1297-308. 
7. Gutiérrez-Fernández M, Rodríguez-Frutos B, Ramos-Cejudo J, et al. Effects of intravenous administration of allogeneic bone marrow- and adipose tissue-derived mesenchymal stem cells on functional recovery and brain repair markers in experimental ischemic stroke. Stem Cell Res Ther 2013, 4:11.

8. Gutiérrez-Fernández M, Rodríguez-Frutos B, Otero-Ortega $L$, et al. Adipose tissuederived stem cells in stroke treatment: from bench to bedside. Discov Med 2013; $16: 37-43$

9. Gutiérrez-Fernández M, Fuentes B, Rodríguez-Frutos B, et al. Trophic factors and cell therapy to stimulate brain repair after ischaemic stroke. J Cell Mol Med 2012; 16:228090

10. Gutiérrez-Fernández M, Rodríguez-Frutos B, Ramos-Cejudo J, et al. Comparison between xenogeneic and allogeneic adipose mesenchymal stem cells in the treatment of acute cerebral infarct. Proof of concept in rats. J Trans Med 2015; 13: 46. * AD-MSC administration was safe and without side effects or tumor formation in experimental animal model of stroke.

11. Ikegame Y, Yamashita K, Hayashi S, et al. Comparison of mesenchymal stem cells from adipose tissue and bone marrow for ischemic stroke therapy. Cytotherapy 2011; 13 : $675-85$.

12. Leu S, Lin YC, Yuen CM, et al. Adipose-derived mesenchymal stem cells markedly attenuate brain infarct size and improve neurological function in rats. J Transl Med 2010; 8: 63 .

13. Lin $\mathrm{XH}$, Liu N, Xiao YC, et al. Effects of adipose-derived stem cells transplantation on the neuronal apoptosis and the expression of $\mathrm{Bcl}-2$ and caspase-12 in the brain post focal cerebral ischemia in rats. Chinese J Cell Mol Immunol 2011; 27:40-3.

14. Erba P, Terenghi G, Kingham PJ. Neural differentiation and therapeutic potential of adipose tissue derived stem cells. Curr Stem Cell Rest 2010; 5:153-60.

15. Wang JH, Liu N, Du HW, et al. Effects of adipose-derived stem cell transplantation on the angiogenesis and the expression of bFGF and VEGF in the brain post focal cerebral ischemia in rats. J Cell Mol Immunol 2008; 24:958-61.

16. Matute C, Domercq M, Pérez-Samartin A, et al. Protecting white matter from stroke injury. Stroke 2013; 44:1204-11.

17. Friedenstein AJ, Deriglasova UF, Kulagina NN, et al. Precursors for fibroblasts in different populations of hematopoietic cells as detected by the in vitro colony assay method. Exp Hematol 1974; 2: 83-92. 
18. Zuk PA, Zhu $M$, Mizuno $H$, et al. Multilineage cells from human adipose tissue: implications for cell-based therapies. Tissue Eng 2001; 7:211-28. *The first evidence showing that human lipoaspirate contains multipotent cells and may represent an alternative stem cell source to bone marrow-derived MSCs.

19. Kang SK, Lee DH, Bae YC, et al. Improvement of neurological deficits by intracerebral transplantation of human adipose tissue-derived stromal cells after cerebral ischemia in rats. Exp Neurol 2003; 183:355-66.

20. Gutiérrez-Fernández M, Rodríguez-Frutos B, Ramos-Cejudo J, et al. Stem cells for brain repair and recovery after stroke. Expert Opin Biol Ther 2013;13:1479-83

21. Melief SM, Zwaginga JJ, Fibbe WE, Roelofs H. Adipose tissue-derived multipotent stromal cells have a higher immunomodulatory capacity than their bone marrowderived counterparts. Stem cells Trans Med 2013; 2:455-63

22. Detante O, Moisan A, Dimastromatteo J, et al. Intravenous administration of 99mTcHMPAO-labeled human mesenchymal stem cells after stroke: in vivo imaging and biodistribution. Cell Transplant 2009; 18: 1369-79.

23. Yang B, Migliati E, Parsha K, et al. Intra-arterial delivery is not superior to intravenous delivery of autologous bone marrow mononuclear cells in acute ischemic stroke. Stroke 2013; 44:3463-72.

24. Fernandez R, Nardocci G, Navarro C, et al. Neural reflex regulation of systemic inflammation: potential new targets for sepsis therapy. Front Physiol 2014; 5:489.

25. Wei X, Du Z, Zhao L, et al. IFATS collection: The conditioned media of adipose stromal cells protect against hypoxia-ischemia-induced brain damage in neonatal rats. Stem Cells 2009; 27:478-88.

26. Cho YJ, Song HS, Bhang S, et al. Therapeutic effects of human adipose stem cellconditioned medium on stroke. J Neurosci Res 2012; 90:1794-802.

27. Hayashi T, Abe K, Itoyama Y. Reduction of ischemic damage by application of vascular endothelial growth factor in rat brain after transient ischemia. J Cereb Blood Flow Metab. 1998; 18:887-95.

28. Jeon D, Chu K, Lee ST, et al. Neuroprotective effect of a cell-free extract derived from human adipose stem cells in experimental stroke models. Neurobiol Dis 2013; 54:41420.

29. Lai RC, Chen TS, Lim SK. Mesenchymal stem cell exosome: a novel stem cell-based therapy for cardiovascular disease. Regen Med 2011; 6: 481-92. 
30. Xin $\mathrm{H}, \mathrm{Li} \mathrm{Y}, \mathrm{Cui} \mathrm{Y}$, et al. Systemic administration of exosomes released from mesenchymal stromal cells promote functional recovery and neurovascular plasticity after stroke in rats. J Cereb Blood Flow Metab 2013; 33:1711-5.

31. Otero L, Bonilla C, Aguayo C, Zurita M, Vaquero J. Intralesional administration of allogeneic bone marrow stromal cells reduces functional deficits after intracerebral hemorrhage. Histol Histopathol 2010; 4:453-61.

32. Otero L, Zurita $\mathrm{M}$, Bonilla $\mathrm{C}$, et al. Late transplantation of allogeneic bone marrow stromal cells improves neurologic deficits subsequent to intracerebral hemorrhage. Cytotherapy 2011; 5:562-71.

33. Otero L, Zurita M, Bonilla $\mathrm{C}$, et al. Allogeneic bone marrow stromal cell transplantation after cerebral hemorrhage achieves cell transdifferentiation and modulates endogenous neurogenesis. Cytotherapy 2012; 14:34-44.

34. Ahn SY, Chang YS, Sung DK, et al. Mesenchymal stem cells prevent hydrocephalus after severe intraventricular hemorrhage. Stroke 2013; 2:497-504.

35. Chen J, Tang YX, Liu YM, et al. Transplantation of adipose-derived stem cells is associated with neural differentiation and functional improvement in a rat model of intracerebral hemorrhage. CNS Neurosci Ther 2012; 10: 847-54.

36. Kim JM, Lee ST, Chu K, et al. Systemic transplantation of human adipose stem cells attenuated cerebral inflammation and degeneration in a hemorrhagic stroke model. Brain Res 2007; 1183:43-50.

37. Lim MH, Ong WK, Sugii S. The current landscape of adipose-derived stem cells in clinical applications. Expert Rev Mol Med 2014; 16: e8. *This review seeks to highlight the clinical applicability of AD-MSC as well as their progress in clinical trials across various medical disciplines.

38. Gao S, Zhao P, Lin C, et al. Differentiation of human adipose-derived stem cells into neuron-like cells which are compatible with photocurable three-dimensional scaffolds. Tissue Eng Part A 2014; 20: 1271-84.

39. Keeney M, Deveza L, Yang F. Programming stem cells for therapeutic angiogenesis using biodegradable polymeric nanoparticles. J Vis Exp 2013; 79:e50736.

40. Moriyama $\mathrm{M}$, Moriyama $\mathrm{H}$, Ueda $\mathrm{A}$, et al. Human adipose tissue-derived multilineage progenitor cells exposed to oxidative stress induce neurite outgrowth in PC12 cells through p38 MAPK signaling. BMC Cell Biol 2012; 13: 21. 
41. Shen CC, Yang YC, Chiao MT, et al. Low-level laser stimulation on adipose-tissuederived stem cell treatments for focal cerebral ischemia in rats. Evid Based Complement Alternat Med 2013; 2013: 594906.

42. Tse, WT, Pendleton JD, Beyer WM, Egalka MC, Guinan EC. Suppression of allogeneic Tcell proliferation by human marrow stromal cells: implications in transplantation. Transplantation 2003; 75: 389-97

43. Javazon EH, Beggs KJ, Flake AW. Mesenchymal stem cells: paradoxes of passaging. Exp Hemato 2004; 32:414-25.

44. Du G, Liu Y, Dang $M$, et al. Comparison of administration routes for adipose-derived stem cells in the treatment of middle cerebral artery occlusion in rats. Acta Histochem. 2014;116:1075-84.

45. Gutiérrez M, Merino JJ, Alonso de Leciñana M, Díez-Tejedor E. Cerebral protection, brain repair, plasticity and cell therapy in ischemic stroke. Cerebrovasc Dis 2009; 27:177-86.

46. Chan TM, Harn HJ, Lin HP, et al. The use of ADSCs as a treatment for chronic stroke. Cell Transplant 2014; 23: 541-7.

47. Shen LH, Li Y, Chen J, Zacharek A, et al. Therapeutic benefit of bone marrow stromal cells administered 1 month after stroke. J Cereb Blood Flow Metab 2007; 27: 6-13.

48. Vasconcelos Dos Santos A, Costa Reis J, Diaz Paredes B, et al. Therapeutic window for treatment of cortical ischemia with bone marrow-derived cells in rats. Brain Res 2010; 1306:149-58.

49. ClinicalTrials.gov - Homepage [htpp//:www.clinicaltrials.gov]

50. Feng $\mathrm{Y}$, Zhu $\mathrm{M}$, Dangelmajer $\mathrm{S}$, et al. Hypoxia-cultured human adipose-derived mesenchymal stem cells are non-oncogenic and have enhanced viability, motility, and tropism to brain cancer. Cell Death Dis 2014; 5:e1567.

51. Wei HJ, Zeng R, Lu JH, et al. Adipose-derived stem cells promote tumor initiation and accelerate tumor growth by interleukin-6 production. Oncotarget 2015; Mar 8. [Epub ahead of print]

52. Freese KE, Kokai L, Edwards RP, et al. Adipose-Derived Stems Cells and Their Role in Human Cancer Development, Growth, Progression, and Metastasis: A Systematic Review. Cancer Res 2015; Mar 3. doi: 10.1158/0008-5472.CAN-14-2744

53. Sozmen EG, Kolekar A, Havton LA, et al. A white matter stroke model in the mouse: axonal damage, progenitor responses and MRI correlates. J Neurosci Meth 2009; 180:261-72. 
54. Rosenzweig S, Carmichael ST. Age-dependent exacerbation of white matter stroke outcomes: a role for oxidative damage and inflammatory mediators. Stroke 2013;44:2579-86.

55. Ramos-Cejudo J, Gutiérrez-Fernández M, Otero-Ortega L, et al. Brain-derived neurotrophic factor administration mediated oligodendrocyte differentiation and myelin formation in subcortical ischemic stroke. Stroke. 2015; 46:221-8. 


\section{Article highlights}

- AD-MSCs are a promising therapy for ischemic and hemorrhagic stroke treatment.

- Many questions are still to be resolved regarding cell production, the route of administration, the therapeutic window, and mechanisms of action and immune modulation.

- AD-MSC production must be optimized according to the desired time to administration in stroke patients.

- It is necessary to evaluate the safety of AD-MSCs in stroke patients.

Legend of figures

Figure 1. Representation of alternatives to AD-MSC-derived treatment production and the time required to obtain them. A) AD-MSCs obtained from a voluntary healthy donor take 1 week for their preparation prior to administration B) Autologous sources need 4 weeks for preparation. C) Continuous production of AD-MSCs would mean 1 day would be needed prior to administration.

Figure 2. Therapeutic strategies mediated by various time points of AD-MSC administration: early or late. Brain protection and repair mechanisms are activated as a continuum after stroke. Both mechanisms are enhanced after early AD-MSC administration, while late AD-MSC administration only enhances brain repair mechanisms. The handicap of early administration is the difficulty in terms of obtaining an adequate number of AD-MSCs. 
Figure 1

A

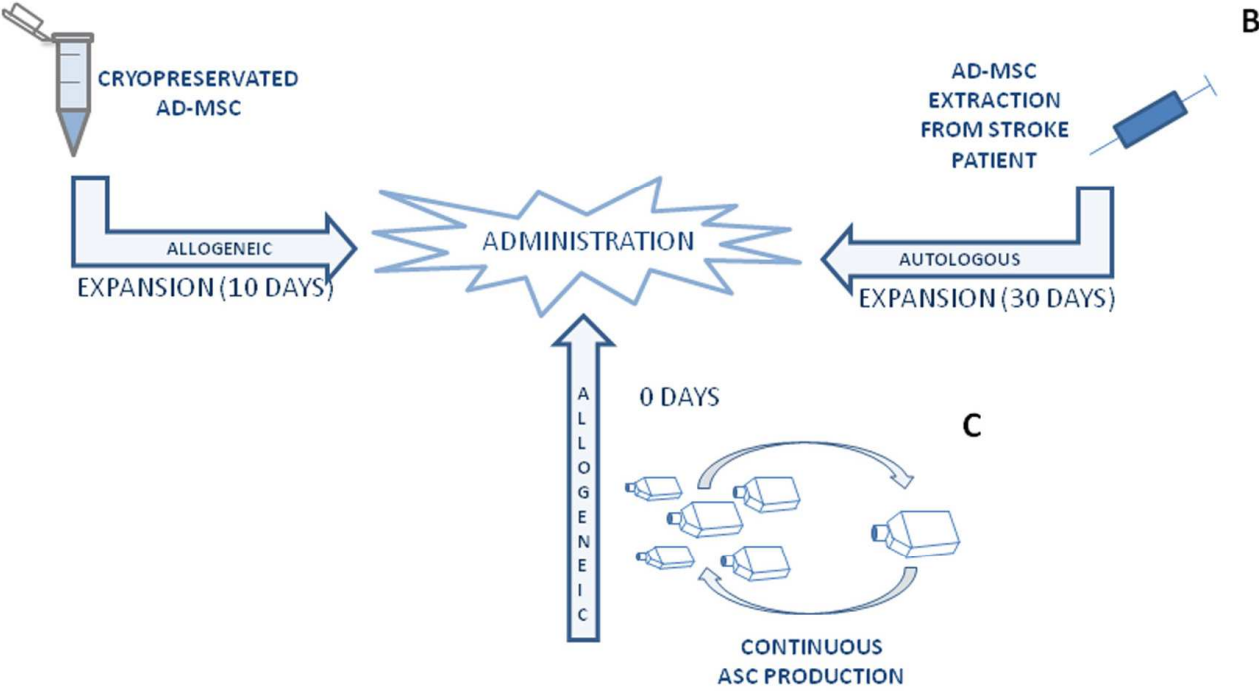




\section{Figure 2}

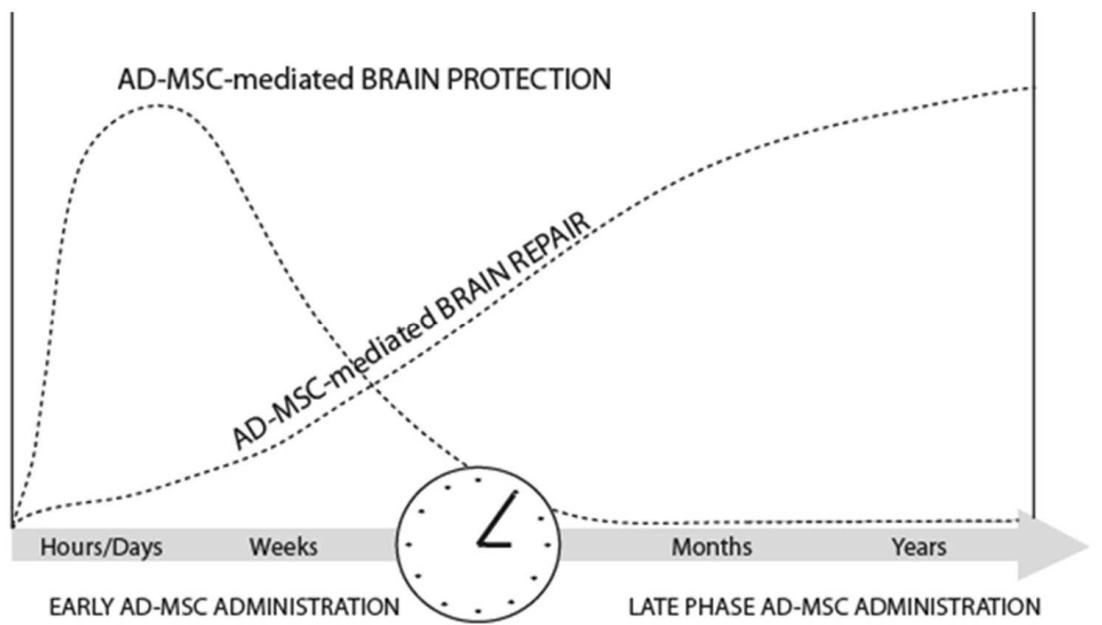

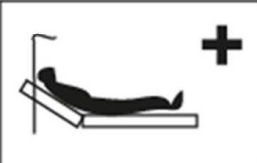

Brain protection and repair are enhanced Difficult to obtain sufficent amounts of AD-MSC

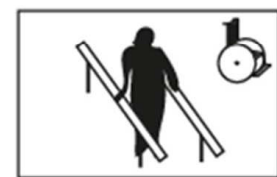

Effects limited to brain repair Easy to obtain sufficent amounts of AD-MSC 